\title{
Digital Memory Look-up based implementation of Sliding Mode Control for DC-DC Converters
}

\author{
Bibaswan Banerjee $^{\mathrm{a}}$, Ramachandra M. Kotecha ${ }^{\mathrm{a}}$, Wayne W. Weaver ${ }^{\mathrm{a}}$ \\ ${ }^{a}$ Michigan Technological University, 1400 Townsend Dr., Houghton, Michigan, 49931, \\ USA
}

\section{Abstract}

Switched power electronic converters involve different control actions for different system events. A local control strategy may be developed which reacts only to some local information available to each component without any communication between the different system components located far away in real time. The purpose of this paper is to present a low cost memory based control strategy in a dc-dc boost converter. The control employed in this work is based on a sliding-mode hysteretic control strategy where the sliding manifold is derived a-priori and stored as a look-up table in digital memory hardware. The proposed control implementation strategy is low cost and offers a robust dynamic response that is used to mitigate many disturbances in the system.

Keywords: Power Electronic Converters, Power Electronics, DC-DC Boost Converter, Digital Control, Sliding Mode Control, Hysteretic control, Predictive control

Email addresses: bbanerje@mtu.edu (Bibaswan Banerjee), rmkotech@mtu.edu (Ramachandra M. Kotecha), wwweaver@mtu.edu (Wayne W. Weaver)

Preprint submitted to Control Engineering Practice

May 6, 2016

(C) 2016. This manuscript version is made available under the Elsevier user license http://www.elsevier.com/open-access/userlicense/1.0/ 


\section{INTRODUCTION}

In modern day smart grids and microgrids, the usage of dc-dc power electronic converters has increased due to the requirement of various levels of dc voltages in home and office facilities [1]. Switching in the power electronics is a non-linear phenomena and application of the traditional linear control techniques are often not suitable [2]. The classical linear control technique has limitations in large signal transients, such as step changes in the load or startup processes [3]. Thus, there is a need for a control technique which is capable of dealing with non linearities and wide variations in load, while ensuring uninterrupted operation and at the same time providing a good transient response $[4,5,6]$. This advanced capability often comes at the price of a complex and costly control hardware [7]. Switching converters form a variable structure system and thus sliding-mode control is an effective and simpler technique [8,9] than other robust control schemes which are computationally intensive $[10,11]$. Previous work has been done on the development of sliding-mode control strategies in power electronics, which drive the system states to some reference $[2,4,12,13,14,15]$. The use of digital control in switching power converters has increased due to the decreased cost and increased computational capability of digital ICs [16, 17, 18]. Some work has been done with the digital implementation of a control strategy by using a predetermined surface inside a digital controller [19, 15].

This work shows a method of implementing a digital sliding-mode hysteretic control technique in a switching power electronic converter. A dc-dc boost converter is chosen to validate the strategy. The stability analysis of various reference surfaces chosen for the controller is performed in this work. 
This work uses [19] as the starting point and implements a digital slidingmode hysteretic control method in a dc-dc boost converter where the sliding surfaces are predetermined and stored in a low-cost memory circuit, instead of using any form of software component, such as a Digital Signal Processor (DSP), in the design. The use of this control strategy eliminates the necessity of any analog tuning and communication. It is also shown that by using analog-to-digital converters (ADC) in the process, the control can be improved by eliminating the need to derive and implement a hysteresis band which limits the effective switching frequency [20]. The inherent propagation delay in the ADC creates the required hysteresis band in the controller. Since the sliding surfaces are derived a-priori, the system response time is reduced.

\section{Implementation of digital hysteretic sliding-mode controller in a dc-dc converter}

The use of digital control in switching power converters has increased due to the decreasing cost of digital controllers, such as microcontrollers, DSPs and Electrically Erasable Programmable Read-Only Memory (EEPROM) [16]. A sliding-mode control surface for power electronics can be implemented in all these devices. However, a EEPROM device has the lowest cost with the best response rate of all the mentioned devices. Different choices of reference surfaces can be calculated a-priori and stored as a memory lookup table in the device. This memory table contains the complete surface and the switching law. An analog-to-digital conversion of the state feedback signals of dc-dc converter are used as the quantized digital addresses of the memory table. The proper switch states (1 or 0 ) are stored in the memory 


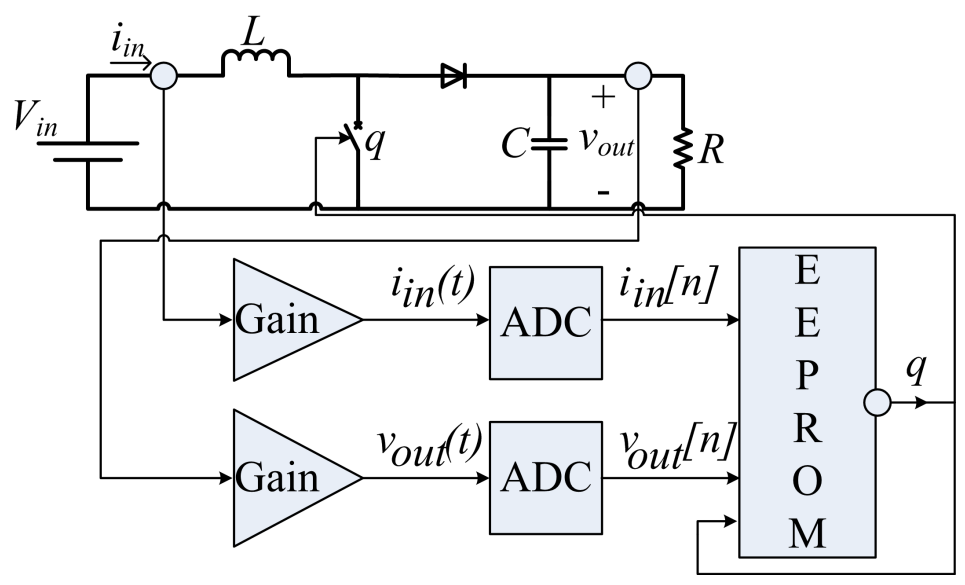

Figure 1: Circuit for the implementation of the digital sliding-mode control strategy with two ADCs and a single EEPROM in a dc-dc boost converter.

location according to the a-priori calculations. A hysteretic band can also be introduced by feeding the current switch state back as an address component for the next switch state. The circuit of a dc-dc boost converter, along with the memory lookup table/EEPROM controller are shown in Fig. 1. The sample times for the ADCs are $2.2 \mu \mathrm{s}, 2.5 \mu \mathrm{s}$ and $2.8 \mu \mathrm{s}$ and these are the times used in the simulations and experiments.

\section{Experimental apparatus}

In Fig. 1 the analog signals from the power stage of the converter are converted into digital signals of $M$ bits using parallel interface ADCs. The digital output of the ADCs are interfaced with a parallel EEPROM chip, which stores the switching surfaces based on the open-loop solutions for different desired response scenarios. The switching surfaces are stored as pixels 
in the address locations of the EEPROM. The output of the EEPROM is the switch state. Corresponding to each address in the memory, a switch state is programmed by an algorithm that compares the pixel for that address to the reference surface and computes the switch states. Therefore, in the EEPROM, the complete surface is stored as different addresses and each address has a switch state associated with it. Thus, the EEPROM forms the digital controller that outputs the switch state based on hysteretic slidingmode control technique. From the two parallel digital devices an entire byte of data is transmitted. Therefore, when information from the analog signal of the power stage is converted to an $M$-bit digital signal at the output of the ADC the entire $M$-bit of data is transmitted to the parallel EEPROM at the same time. The physical address space is a $p$-dimensional look-up table (where $p$ is the number of analog signals being fed into the ADC converters). Therefore, in Fig. $1, i_{i n}$, the input current, and $v_{\text {out }}$, the output voltage, are M-dimensional vectors with binary entries. This control scheme has a very fast response time when the ADCs with appropriate resolution and fast sampling rate EEPROMs are used. For implementing the hysteretic sliding-mode control of the power converters, an 8 bit ADC is used for each analog signal. This is a generic hardware set-up that can be used to control a switching power converter with any number of state-analog signals. The gain blocks shown in Fig. 1 are voltage dividers that step down the state analog signals to appropriate levels for the ADCs. This will depend on the maximum input voltage limit of the ADCs. 


\section{Memory resolution analysis}

The dc-dc boost converter depicted in Fig. 1 is based on the assumption of an ideal switch and diode to the dynamic system equations

$$
\begin{aligned}
& L \dot{i}_{\text {in }}=V_{\text {in }}-(1-q) v_{\text {out }} \\
& C \dot{v}_{\text {out }}=(1-q) i_{\text {in }}-\frac{v_{\text {out }}}{R},
\end{aligned}
$$

where $i_{\text {in }}$ is the input current, $v_{\text {out }}$ is the output voltage, $V_{\text {in }}$ is the input voltage, $q$ is the switch state, $L$ and $C$ are energy storage elements and $R$ is a constant resistive load. An ideal switch for the dc-dc converter is considered for mathematical modeling and simulation. The state space representation of (1) gives

$$
\dot{x}(t)=\left[\begin{array}{cc}
0 & -\frac{1-q}{L} \\
\frac{1-q}{C} & -\frac{1}{C R}
\end{array}\right] x(t)+\left[\begin{array}{c}
\frac{V_{i n}}{L} \\
0
\end{array}\right]
$$

where

$$
x(t)=\left[\begin{array}{c}
i_{\text {in }}(t) \\
v_{\text {out }}(t)
\end{array}\right] .
$$

The system parameters and the states are combined into $\lambda=\left[R, L, C, V_{i n}\right]^{\top}$ and $x(t)=\left[i_{\text {in }}, v_{\text {out }}\right]^{\top}$ respectively. The switch state $q$ is generated from a digital hysteretic sliding-mode controller. The system state trajectories are first attracted towards a reference sliding surface given by

$$
s=0
$$

based on the switching control law

$$
q= \begin{cases}0 & \text { if } s-\frac{h}{2}>0 \\ 1 & \text { if } s+\frac{h}{2}<0 \\ q & \text { else. }\end{cases}
$$


Then they travel along $s$ and finally limit cycles about the intersection of $s=0$ and a desired steady state operating point $x_{e}=\left[i_{\text {inop }}, v_{o p}\right]^{\top}$, within a band $h>0$ known as the hysteresis band.

A dc-dc boost converter has a range of operating points depending on $\lambda$ and $x(t)$. The load line results from the steady state condition of the system shown in (2) and can be given by

$$
i_{\text {in }}=\frac{v_{o u t}^{2}}{R V_{\text {in }}}
$$

The sliding surface chosen for this analysis is a sloped linear surface in the state-plane. The reason for the choice of this surface is discussed in Section 6.2. The equation for the surface is

$$
s=i_{\text {in }}-m+b v_{\text {out }}
$$

where $m$ is the $i_{i n}$-axis intercept and $b$ is the slope of the line. The slope of the line is determined by

$$
b=\frac{m}{v_{o p}}-\frac{v_{o p}}{R V_{i n}}
$$

where $v_{o p}$ is the desired voltage operating point. The intersection of $s$ and the load line determines the steady state operating point around which the states limit cycle.

The surface shown in (7) is a function of time and the states, assuming the $i_{\text {in }}$ intercept constant. The surface is also a function of system parameters $\lambda$, since the slope $b$ is dependent on $R$. The surface can be constructed a-priori in a memory lookup table with open-form scaled state values in the form of a 


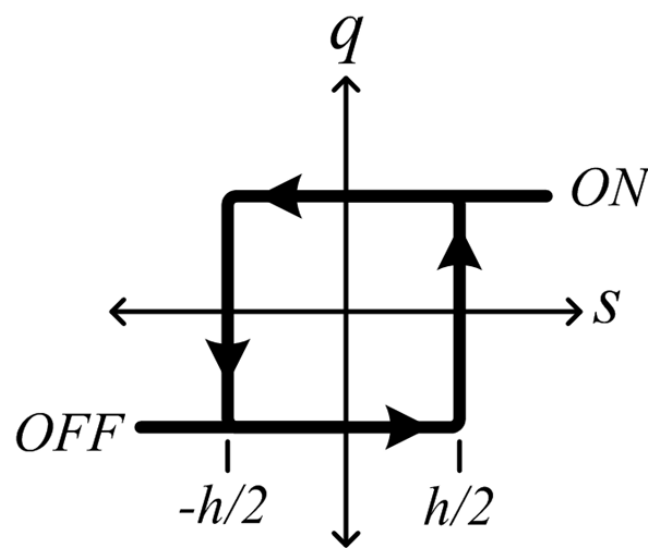

Figure 2: Figure illustrating the generation of the hysteresis band.

table called raster surface. In the system shown in Fig. 1, the ADCs convert the state signals $x(t)$ into sampled signals $x[n]$. The sampling resolution steps depend on the number of bits of the ADC, e.g. if the number of ADC bits is $M$, the number of resolution steps is $2^{M}$. The number of resolution steps gives the number of memory addresses needed for the surface generation. The sampled signals $x[n]$ form the pixels of the surface. The complete surface is generated by many such pixels taken together. This surface can be further divided into two separate regions based on the switch state. The pixels are compared to (7) and depending on whether they are greater than or less than zero, form the $s>0(\mathrm{OFF})$ and $s<0(\mathrm{ON})$ regions. The surface generated so far does not include the hysteresis bands in it. To generate the hysteresis bands, the switch state feedback to the memory table, as shown in Fig. 1, is necessary. Fig. 2 shows how a hysteresis band is generated using the switch state feedback. If the switch state is $0(\mathrm{OFF})$, the pixels are compared to $s+\frac{h}{2}$. If $s+\frac{h}{2}<0$, it remains $O F F$ and turns $O N$ if $s+\frac{h}{2}>0$. If the 
switch state is $1(\mathrm{ON})$, the pixels are compared to $s-\frac{h}{2}$. If $s-\frac{h}{2}>0$, it remains $O N$ and turns $O F F$ if $s-\frac{h}{2}<0$. Hence, there is a region where the switch state may be $O N$ or $O F F$ depending on the present switch state. This region forms the required hysteresis band. Fig. 4 shows the full surface in the memory comprised of all the pixels. The light pixels form the $O N$ region, the darker pixels form the $O F F$ region, and the region shown by the darkest pixels, is the hysteresis band. These pixels form the addresses of the memory lookup table.

The output of the memory lookup table is the switch state. Corresponding to each address in the memory, a switch state is programmed by an algorithm that compares the pixel for that address to the surface (7) and computes $q$ based on (5). A simulation is done in MATLAB/Simulink for the system shown in Fig. 1 with system parameters shown in Table 1. The system sensor states are sampled in the ADCs. The sampled states from the ADC lookup the switch state from the memory table. This switch state controls the switch of the dc-dc boost converter and drives the system states from a cold start to the desired equilibrium point.

Fig. 3 and Fig. 4 show simulation results for two different resolution steps. Fig. 3 depicts the result for a resolution step size of 16 per state variable. Here only 4 bits of each ADC are used which leads to 256 address locations of the memory lookup table. A very low resolution step value implies low number of pixels in the hysteresis band. The system states have to lookup for the correct switch state from only 256 address locations. This results in improper switch states and the controller is unable to drive the system states to the surface and take them to the desired equilibrium point. In this 


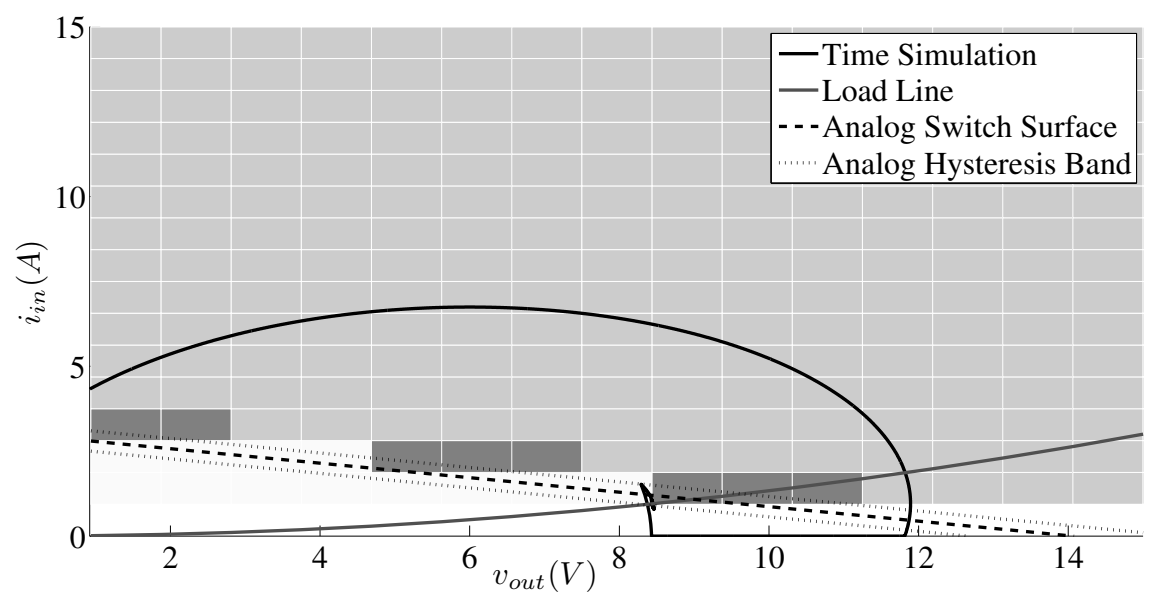

Figure 3: State-plane plot for the digital sliding-mode control strategy for a sloped reference surface showing the memory resolution effect for a resolution step size of 16 .

case, the pixels in the hysteresis band are not a good representation of the actual hysteresis band. Fig. 4 shows the simulation result for a resolution step size of 128 per variable. Here, 7 ADC output bits and 16384 memory locations of the chip are used. It may be noted that as the resolution step per state variable is increased, the number of pixels in the hysteresis band rises and gives a better representation of the actual hysteresis band as well as better control. Fig. 4 shows that the system state trajectories are effectively attracted to the quantized surface and driven to the desired equilibrium point.

Table 1: Example system parameters for a sloped linear reference surface control.

\begin{tabular}{|c|c|c|c|c|c|c|c|c|}
\hline$V_{i n}$ & $L$ & $C$ & $R$ & Memory resolution step & $m$ & $b$ & $v_{o p}$ & $h$ \\
\hline $6 \mathrm{~V}$ & $1 \mathrm{mH}$ & $100 \mu \mathrm{F}$ & $12.5 \Omega$ & $16 / 128$ & $3 \mathrm{~A}$ & $0.213 \mathrm{~A} / \mathrm{V}$ & $9 \mathrm{~V}$ & $0.6 \mathrm{~A}$ \\
\hline
\end{tabular}




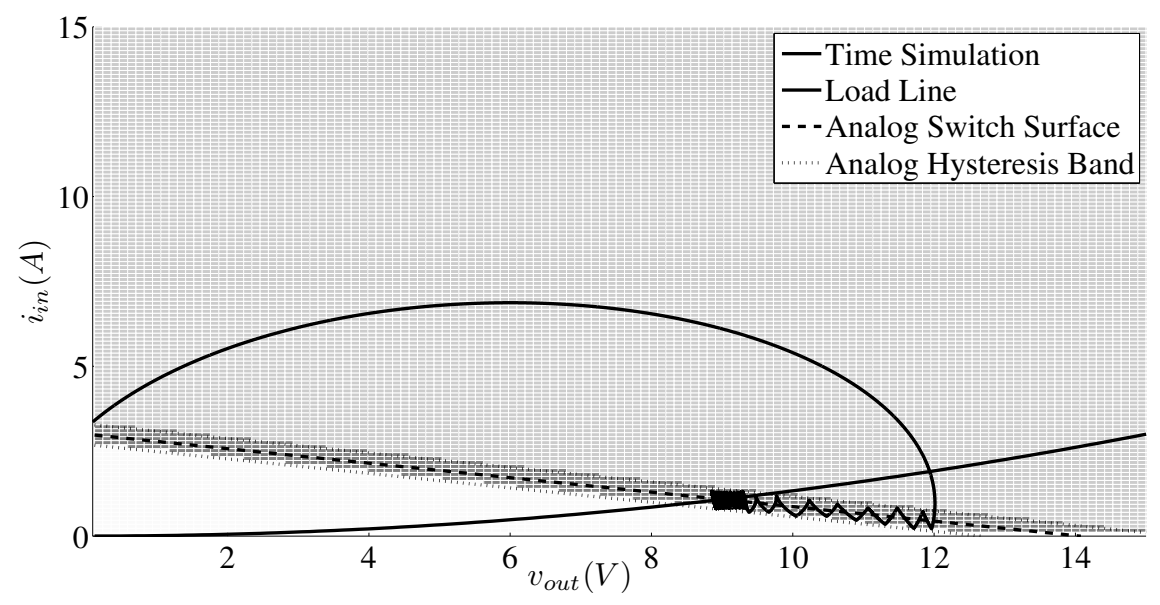

Figure 4: State-plane plot for the digital sliding-mode control strategy for a sloped reference surface showing the memory resolution effect for a resolution step size of 128 .

The shape of the surface and the operating point depend on the system parameters that may change during operation. Possible changes in the circuit parameters may be taken into account during the process of surface generation in the memory lookup table. The system used for simulation has a $R$ value of $12.5 \Omega$. If there is a step change in the load resistance, the surface and the load line both will change, hence the equilibrium point will also change. It is necessary to construct this new surface in the memory lookup table. This will require additional memory locations to accommodate the additional pixels for this surface and the switch states corresponding to them. The memory lookup table will require sensing the resistance values for the correct lookup for the switch state. Similarly, changes in other system parameters will require more memory locations in the lookup table. It is possible to derive and store many surfaces for many different system parameters a-priori and generate the required switch states for the control. 


\section{Control hardware cost comparison}

Implementation of a digital sliding-mode control technique in dc-dc boost converters requires a complex controller as discussed in Sections 3 and 4. A high-end digital signal processor or an advanced microcontroller is required to implement complex sliding surfaces in the form of a look-up table in the controller. In this work, the memory table and the control logic are programmed in an EEPROM (ATMEL Part Number AT28C256). A Virtex5 FPGA platform is used together with dSPACE DS1104 to implement a sliding-mode control strategy in a boost converter for fuel cell applications in [21]. However, this is a development tool and hence not included in the price comparison. The work done in [19] used a DSP (Part Number TMS320F2812) with a link for computer interface (Code Composer Studio). A 32 bit microcontroller (LPC1768 32 bit ARM Cortex-M3) along with a RTII ethernet connection is used in [22] to implement bang-bang control in dc-dc boost converters. The work in [23] used the TMDSCNCD28335 - TMS320F28335 controlCARD from Texas Instruments to implement an adaptive hysteresis band control method in an active power filter application for non sinusoidal supply voltages. A simple PI controller is applicable to constant reference surfaces but it cannot be applied to more complex surfaces, such as a linear

sloped surface shown in Fig. 14. As of April 16, 2016, the prices for all the controllers are compared and shown in Table $2[24,25]$. Therefore, the EEPROM that is used for this work is one of the low cost options available that is capable of performing a similar type of task. 
Table 2: Cost comparison between different digital controllers capable of implementing digital sliding-mode control strategy in a dc-dc boost converter.

\begin{tabular}{|l|c|}
\hline Controller & Price per unit \\
\hline TMS320F2812 DSP & $\$ 29.93$ \\
\hline LPC1768 32-bit ARM Cortex-M3 & $\$ 17.45$ \\
Microcontroller Unit with RMII & \\
Ethernet transceiver & \\
\hline $\begin{array}{l}\text { TMDSCNCD28335 } \\
\text { TMS320F28335 controlCARD }\end{array}$ & $\$ 69.00$ \\
\hline $\begin{array}{l}\text { AT28C256 EEPROM with } \\
\text { LTC1099CN ADC }\end{array}$ & $\$ 17.40$ \\
\hline
\end{tabular}

\section{Simulation and hardware results}

Two different sliding surfaces are chosen to validate the proposed control strategy. A constant current reference surface is selected at first, which is a straight line in the state-plane diagram [26]. The state-plane diagram has the output voltage state in the $\mathrm{x}$-axis and the input current state along the y-axis. Next, a sloped linear surface in the state-plane diagram is chosen. The reason for the choice of these surfaces as well as the stability issues are discussed in the following sections.

\subsection{Linear straight line surface}

A constant current reference surface is selected to validate the control strategy. The goal is to drive the input current state of a dc-dc boost converter from a cold start to the surface and stay on it. The dc-dc boost converter acts as a Point-of-load converter (POLC) between a dc source and 
a resistive load. Since the goal is to control the input current state only, a single 8 bit ADC is used. For the experimental design, the least significant bit (LSB) of the ADC output is not used because of noise issues. Effectively 7 output bits of the ADC are interfaced with the EEPROM, thereby yielding a resolution of $38 \mathrm{~mA} /$ bit. For this set up, 128 memory locations of the EEPROM are used where the switch states are programmed. The EEPROM reads in the ADC output and generates the switch states as stored in its memory to drive the system state to the desired sliding surface.

In Section 4, it was shown that the hysteresis band in the sliding-mode controller is developed using a switch state feedback to the memory lookup table. This adds to hardware complication where additional memory space is necessary to accommodate the switch state feedback. In a real-time system, an ADC is used in conjunction with a memory chip. There is an inherent signal propagation delay associated with the use of an ADC, which generates the required hysteresis in the sliding-mode controller [20]. Fig. 5 illustrates how hysteresis is generated by a time delay in a signal. An example signal, shown as 'Actual signal' in Fig. 5 is delayed by $\Delta t=0.5 \mathrm{~s}$. The delayed signal is shown as 'Time delayed signal' in Fig. 5. For a power electronic converter this signal is a state, such as current or voltage. When the time delayed signal reaches the sliding reference surface, according to the switching condition for a pure sliding-mode control shown in (9), the state trajectory reverses its path.

$$
q= \begin{cases}0 & \text { if } s \geq 0 \\ 1 & \text { if } s<0\end{cases}
$$

But at this point, the actual signal is at a position higher than the reference 


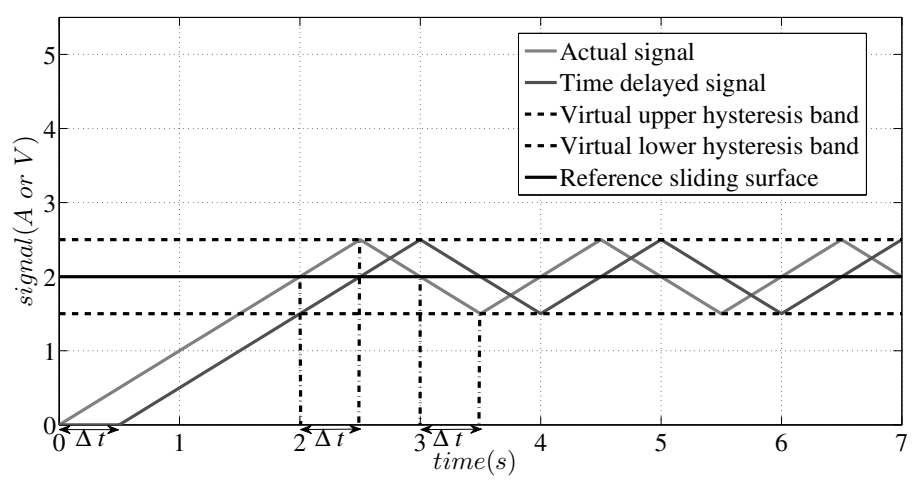

Figure 5: Illustration of hysteresis generation due to time delay of a signal.

surface in the time domain plot as shown in Fig. 5. Again, when the time delayed signal reaches the sliding surface on its return path, the switching takes place according to (9), and the state trajectory reverses its path. This time the actual signal is at a position lower than the reference surface in the time domain plot. This creates a virtual upper and a lower band around the reference surface, where the actual signal changes its path at every switching instant. This band is called the hysteresis band, between which the switching occurs. Therefore, a time delay in the signal can generate the hysteresis required in a sliding-mode controller. This ADC latency therefore, simplifies the hardware complications by reducing the address space in the memory lookup table. There is no need to use additional memory locations for hysteresis generation. The analysis and impact of ADC latency on hysteresis is shown in the following sections.

\subsubsection{Stability analysis for the straight line surface}

For the states of a system to follow a particular sliding surface, the state trajectories need to be attracted towards the manifold. The sliding surface, 
therefore, needs to be a stable one for the system under consideration. It is therefore necessary to prove that the straight line surface chosen for a dc-dc boost converter is a stable surface and the state trajectories are attracted to it.

The dynamic equations for a dc-dc boost converter as shown in Fig. 1 are shown in (1). The switch state for the dc-dc boost converter is determined by the sliding control law

$$
s=f(x)=0
$$

where $x$ represents the converter states and $f(x)$ is the deviation of the states from the reference surface. The switching control law for the dc-dc boost converter is shown in (9). For a system to follow a particular sliding surface, the state trajectories are attracted towards the manifold when

$$
s \dot{s}<0 .
$$

Equation (11) is the required condition for stability [27, 28]. If (11) is satisfied for a region of the state space that contains the sliding surface (10), then the state trajectories that leave any point in this region will converge to the sliding surface and once they reach it, they will stay on it [29]. The straight line surface chosen is defined as

$$
s=i_{\text {in }}-i_{\text {inref }}=0
$$

The derivative of the surface is given by,

$$
\dot{s}=\dot{i}_{\text {in }}=\frac{V_{\text {in }}}{L}-\frac{(1-q) v_{\text {out }}}{L} .
$$

Using (9) in (13), for the region $s<0$,

$$
\dot{s}=\frac{V_{i n}}{L} .
$$


For a dc-dc boost converter, and for any power converter in general, the input voltage and the inductance is always positive. For the example system shown in Table 3, the right hand side of (14) is positive. Therefore, for the region $s<0$,

$$
s \dot{s}<0 .
$$

For the region $s>0$,

$$
\dot{s}=\frac{V_{\text {in }}}{L}-\frac{v_{\text {out }}}{L} .
$$

In the region $s>0$, for stability, $v_{\text {out }}>V_{\text {in }}$. This is the required condition for stability. This condition is always fulfilled for a continuous current mode and therefore there is no limitation. The example dc-dc boost converter system is given a cold start. The initial states $x(0)=[0,0]^{\top}$ lie in the region $s<0$. It has already been proved that all the states lying in the region $s<0$ will slide to the surface. As an illustration, Fig. 6 shows the phase portrait for the example dc-dc boost converter for the region of operation of the system. The phase portrait shows that all the state trajectories are attracted towards the surface and if any of them leave the surface, they again slide back to the surface obeying the switching control law shown in (9). Therefore it may be concluded that the sliding surface under consideration for this dc-dc boost converter system is stable and the states are attracted to the surface.

\subsubsection{Simulation results for the straight line reference surface}

A simulation of the system described above is done in MATLAB/Simulink. The system parameters selected are shown in Table 3. The objective is to drive the input current state trajectory to the constant current reference surface. The final desired input current value is $2.4 \mathrm{~A}$. The ADC delay is $2.5 \mu \mathrm{s}$, 


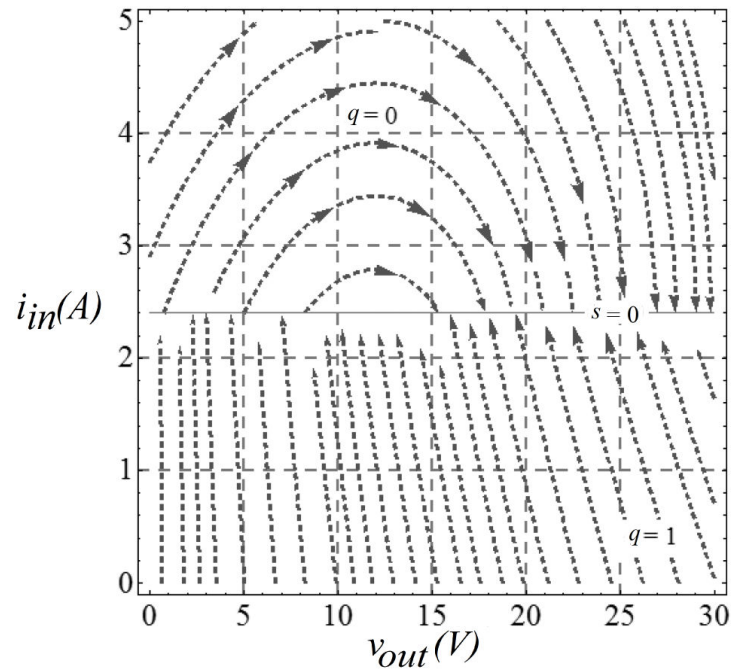

Figure 6: Phase portrait of dc-dc boost converter states for the straight line reference sliding surface.

which generates the hysteresis required by the controller. The simulation results are shown in Fig. 7 and Fig. 8. Fig. 7 illustrates the variation of the input current and output voltage states over time. It may be noted that the input current reaches a value of $2.4 \mathrm{~A}$ from a cold start thereby validating the control strategy. Fig. 8 depicts the state-plane plot of the output voltage and input current states along with the reference sliding surface. The control strategy drives the state trajectories to the surface and compels the states to stay on the surface.

Table 3: Example system parameters for a straight line surface control.

\begin{tabular}{|c|c|c|c|c|c|c|}
\hline$V_{\text {in }}$ & $L$ & $C$ & $R_{\text {load }}$ & ADC resolution & ADC delay & Memory locations \\
\hline $12 \mathrm{~V}$ & $1 \mathrm{mH}$ & $100 \mu \mathrm{F}$ & $12.5 \Omega$ & $38 \mathrm{~mA} / \mathrm{bit}$ & $2.5 \mu \mathrm{s}$ & 128 \\
\hline
\end{tabular}




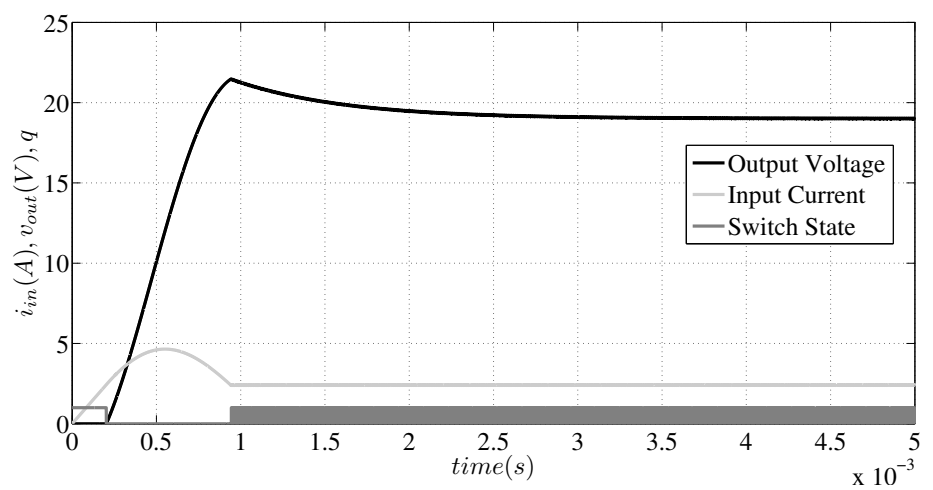

Figure 7: Time domain plot for the digital sliding-mode control strategy for a straight line surface in a dc-dc boost converter.

\subsubsection{Experimental results for the straight line reference surface}

An experimental hardware set up is performed with the same system parameters as in Table 3. The straight line surface is programmed into 128 memory locations of the EEPROM and the ADC is set for a propagation delay of $2.5 \mu \mathrm{s}$. The dc-dc boost converter is constructed in a flexible power electronics drive board. Fig. 9 shows the variation of the input current, output voltage and switch states over time. It may be noted that the input current reaches a value of $2.4 \mathrm{~A}$ from a cold start, thereby experimentally validating the control strategy. Fig. 10 shows the state trajectories in the steady state, indicating that they have reached the reference surface at a value of $2.4 \mathrm{~A}$. Hence, the straight line surface is a stable surface and is used as a reference surface in the controller. The digital sliding-mode hysteretic controller could be effectively designed such that the states reach the reference surface and slide along it. The current limit for the power supply is 5.8 


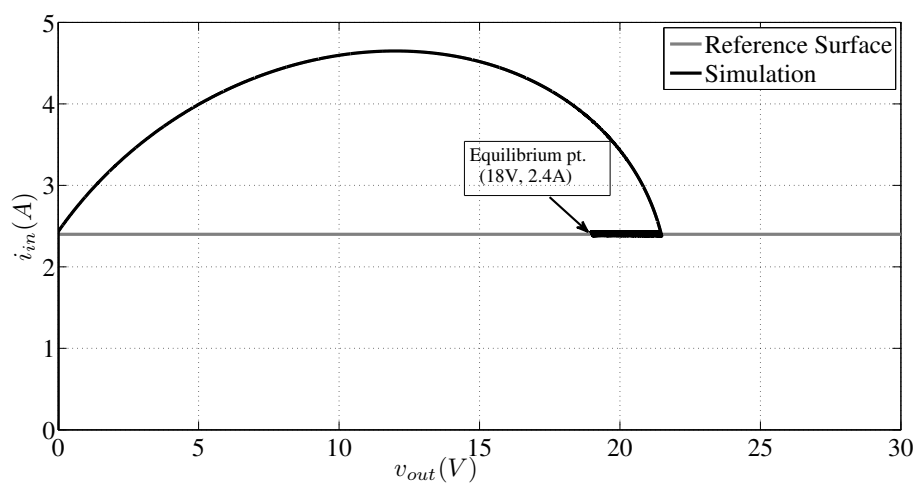

Figure 8: State-plane plot for the digital sliding-mode control strategy for a straight line surface in a dc-dc boost converter.

A. Hence, the experimental results and the simulation results look different. If the current limit could be increased or if a more powerful source could be used, the simulation results would have matched the experimental results. This is a hardware limitation for this work.

\subsubsection{Analysis of the ADC latency and switching frequency for the straight line reference surface}

The simulation and experimental results in the previous subsection confirm that no additional hardware configuration is necessary to generate the required hysteresis band in the digital controller. Instead, the ADC latency is capable of generating this required hysteresis. If the delay time is increased, the hysteresis will increase and vice versa, as evident from Fig. 5. The ADC used for the experimental configuration has three signal propagation delay settings. The default setting is $2.5 \mu \mathrm{s}$, and two extreme settings of $2.2 \mu \mathrm{s}$. and $2.8 \mu \mathrm{s}$. Fig. 11 shows the hysteresis generated due to the default setting 


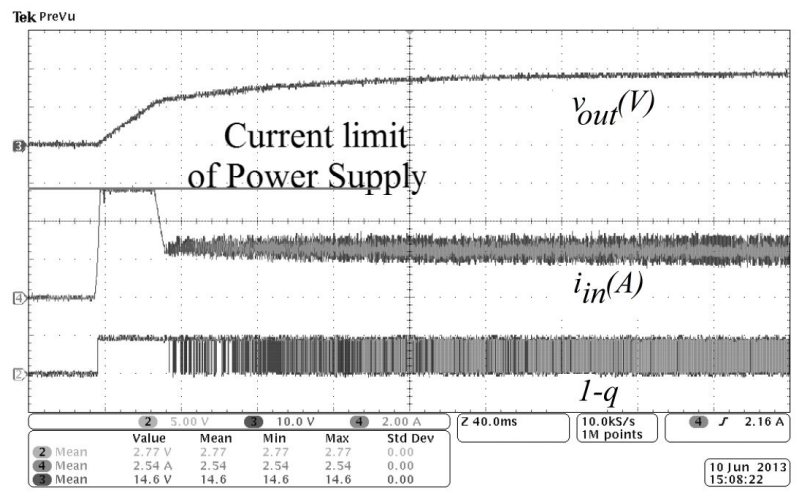

Figure 9: Experimental time domain plot for the digital sliding-mode control strategy for a straight line surface in a dc-dc boost converter. Scale Factors: $10 \mathrm{~V} /$ div for $v_{\text {out }}, 5 \mathrm{~V} /$ div for 1-q, $2 \mathrm{~A} / \mathrm{div}$ for $i_{\text {in }}$ and $40 \mathrm{~ms} / \mathrm{div}$ for the time scale

of $2.5 \mu \mathrm{s}$. conversion delay. Fig. 12 shows the experimental results for a smaller delay of $2.2 \mu \mathrm{s}$. and Fig. 13 shows the results for a greater delay of $2.8 \mu$ s. It may be noted from the results that greater the delay, the larger will be the hysteresis band and lower will be the effective switching frequency. Conversely, narrowing of the band will increase the effective switching frequency. However, there are both upper and lower bounds to the delay in the ADC, beyond which this strategy will cease to work. For this work, the switching frequency is not specified and is directly controlled by the ADC delay. The derivation of a possible mathematical relationship between the $\mathrm{ADC}$ latency and the hysteresis band, and hence the switching frequency, has been left for future work. 


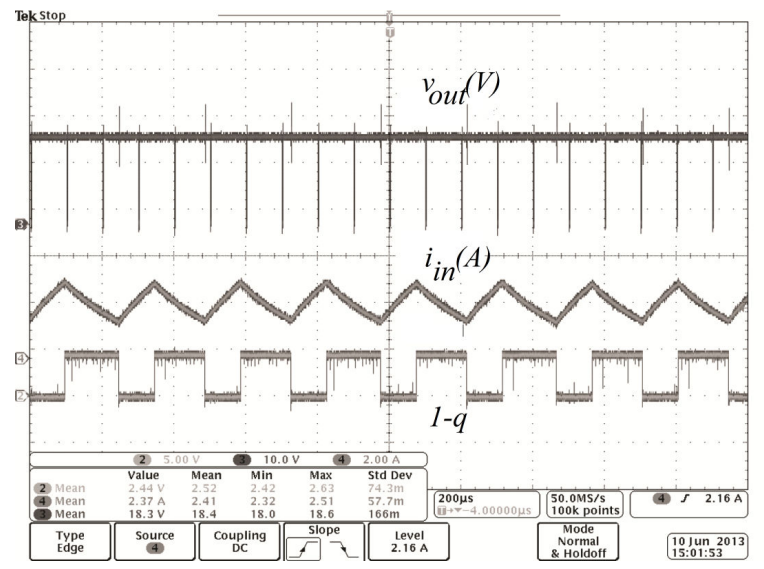

Figure 10: Experimental time domain plot in the steady state for the digital sliding-mode control strategy for a straight line surface in a dc-dc boost converter. Scale Factors: 10 $\mathrm{V} /$ div for $v_{\text {out }}, 5 \mathrm{~V} /$ div for 1 -q, $2 \mathrm{~A} /$ div for $i_{\text {in }}$ and $200 \mu \mathrm{s} /$ div for the time scale 6.2. Linear sloped surface

A linear sloped surface shown in Fig. 14 is next chosen to further validate the proposed control strategy. Any practical reference surface is a non-linear surface in the state-plane diagram. However, a number of sloped linear approximations to any complex non-linear surface may be made for real-time implementation. The choice of a sloped straight line reference surface is therefore pertinent here. The controller designed for this type of a sloped reference surface is a guideline for any type of linear reference surface. The slope and the y-intercept depend on the system design specifications. The sloped line in the state-plane plot is given by

$$
i_{\text {in }}=m-b v_{\text {out }}
$$

The slope of the line is determined by (8). The load line for a dc-dc boost converter is given by (6). Based on the values shown in Table 1, the desired equilibrium point in the state-plane plot is $(9 \mathrm{~V}, 1.08 \mathrm{~A})$. The aim of the 


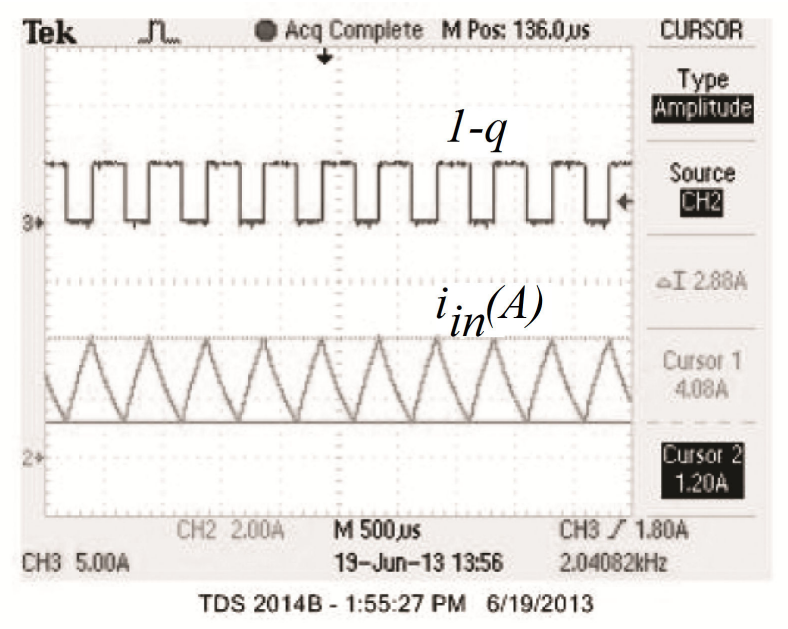

Figure 11: ADC delay of $2.5 \mu$ s generating a hysteresis band of $2.88 \mathrm{~A}$ for the digital sliding-mode control strategy for a straight line surface in a dc-dc boost converter.

controller is to drive the input current and the output voltage states of the dc-dc boost converter from a cold start to slide to the reference surface and finally to reach the desired equilibrium point on the surface. The equilibrium point is defined by the intersection of the load line and the surface. Since the goal here is to control both the input current and output voltage states, two 8 bit ADCs are used. In this set up also, the LSB of the ADC output is not used for noise issues. Effectively 7 output bits of each of the ADC are interfaced with the EEPROM. The resolution for the output voltage is $0.2 \mathrm{~V} /$ bit and that of the input current is $38 \mathrm{~mA} /$ bit. For this set up, $2^{14}=16384$ memory locations of the EEPROM are used where the switch states are programmed. The EEPROM reads in the ADC output and generates the switch states as stored in its memory to drive the system state to the desired equilibrium point on the sliding surface. 


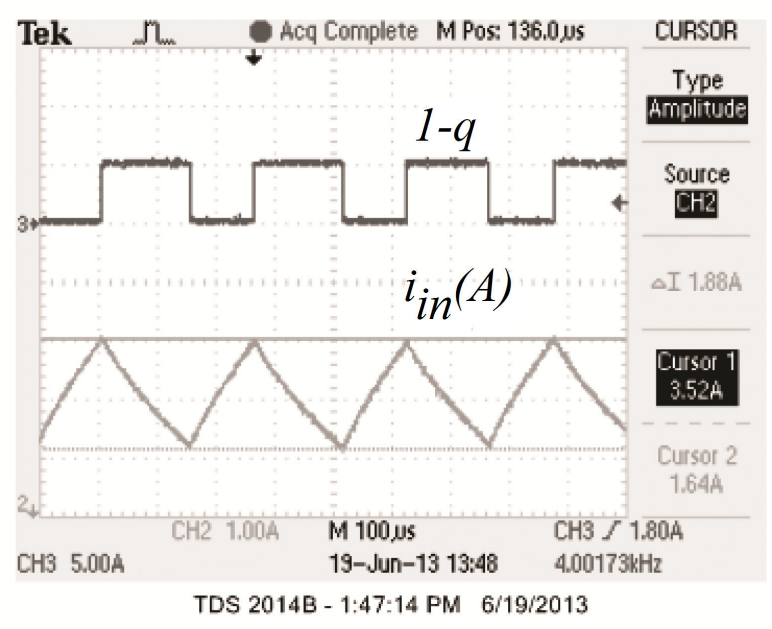

Figure 12: ADC delay of $2.2 \mu$ s generating a hysteresis band of $1.88 \mathrm{~A}$ for the digital sliding-mode control strategy for a straight line surface in a dc-dc boost converter.

\subsubsection{Stability analysis for the linear sloped surface}

The linear approximation of any non-linear surface generates a sloped surface in the state-plane. The sloped linear sliding surface needs to be a stable one for the system under consideration. It is therefore necessary to prove that the sloped linear surface chosen for a dc-dc boost converter is a stable surface. This derivation will generate the condition of stability for any sloped linear surface and then show that the sloped surface for the example system considered is stable.

The dynamic equations for a dc-dc boost converter are shown in (1). The switching control law for the dc-dc boost converter is shown in (9). Equation (11) is the required condition for stability. A sloped linear surface is shown in (7). The derivative of the surface is given by,

$$
\dot{s}=\dot{i}_{\text {in }}-b \dot{v}_{\text {out }} .
$$




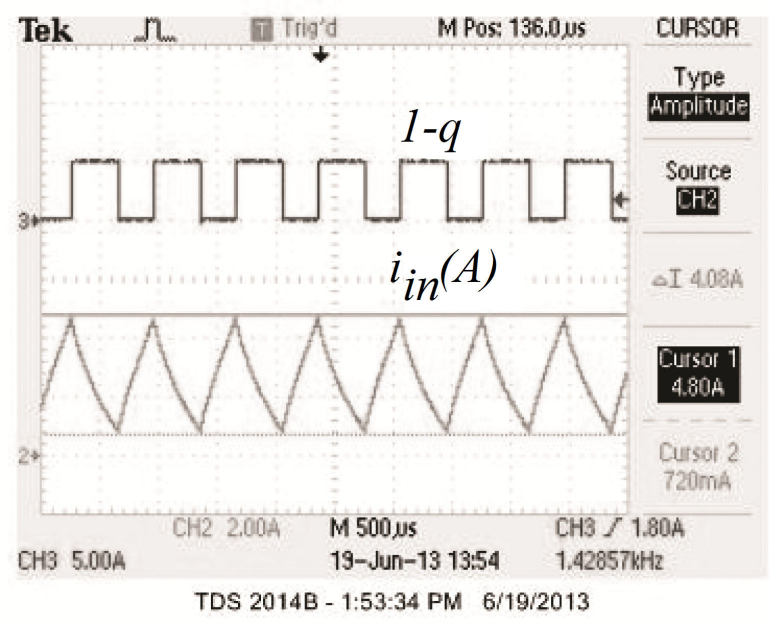

Figure 13: ADC delay of $2.8 \mu$ s generating a hysteresis band of $4.08 \mathrm{~A}$ for the digital sliding-mode control strategy for a straight line surface in a dc-dc boost converter.

Using (9) in (18), for the region $s<0$,

$$
\dot{s}=\frac{V_{\text {in }}}{L}+\frac{b v_{\text {out }}}{C R}
$$

Therefore, for the region $s<0, \dot{s}>0$ when

$$
\frac{V_{\text {in }}}{L}>-\frac{b v_{\text {out }}}{C R}
$$

which is the required stability criteria for this region. For the region $s>0$,

$$
\dot{s}=\frac{V_{\text {in }}}{L}-\frac{v_{\text {out }}}{L}-\frac{b i_{\text {in }}}{C}+\frac{b v_{\text {out }}}{C R} .
$$

For the region $s>0, \dot{s}<0$ when

$$
\frac{V_{\text {in }}}{L}<\frac{C R v_{\text {out }}-L b v_{\text {out }}}{L C R}+\frac{b i_{\text {in }}}{C}
$$

For the example dc-dc boost converter considered for this analysis, the system parameters are shown in Table 1 . Using these parameters, for $s<0$, (19) 


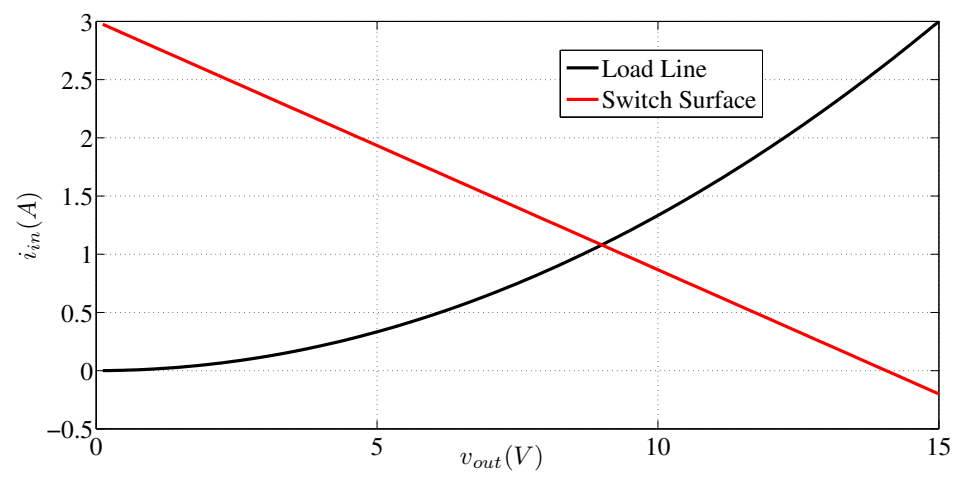

Figure 14: Sloped surface in the state-plane plot for the digital sliding-mode control strategy in a dc-dc boost converter.

reduces to

$$
\dot{s}=6000-170.4 v_{\text {out }} .
$$

For $\dot{s}>0$, the maximum value that the output voltage can take is $35.2 \mathrm{~V}$. Fig. 14 shows that for the region $s<0$, the maximum value of the output voltage is $14 \mathrm{~V}$. Therefore, for the example system, $\dot{s}>0$ for the region $s<0$. Hence, the product is always $s \dot{s}<0$. For the region $s>0,(21)$ reduces to

$$
\dot{s}=2130\left(\frac{v_{\text {out }}}{R}-i_{\text {in }}\right)+1000\left(V_{\text {in }}-v_{\text {out }}\right) .
$$

For stability, in the region $s>0,2130\left(\frac{v_{\text {out }}}{R}-i_{\text {in }}\right)+1000\left(V_{\text {in }}-v_{\text {out }}\right)<0$. This is the required stability criteria. The example dc-dc boost converter system is given a cold start. Both the states are at $(0,0)$ at this point and belong to the region $s<0$. It has already been proved that $\dot{s}>0$ for the region $s<0$ for the example system. Therefore, the states will reach the surface from a cold start. The phase portrait for the example dc-dc boost converter for a region around the sliding surface is shown in Fig. 15. The phase portrait shows that if the states leave the surface, they again slide back 


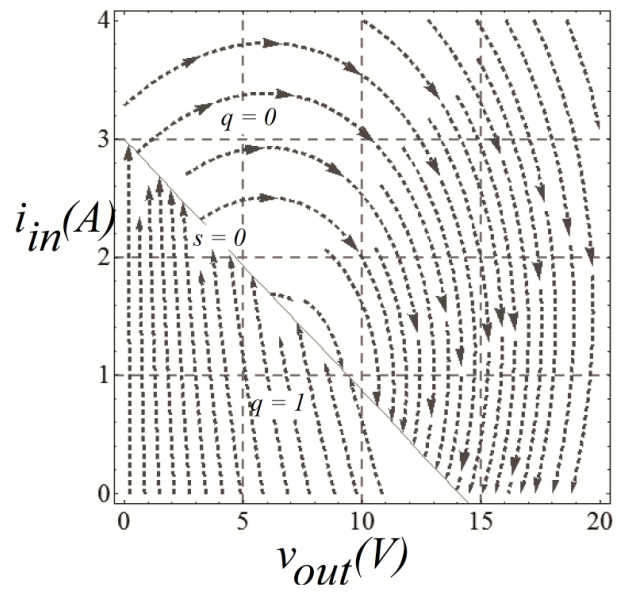

Figure 15: Phase portrait of dc-dc boost converter states for the sloped reference sliding surface.

to the surface obeying the switching control law shown in (9). Therefore it may be concluded that the sliding surface under consideration for this dc-dc boost converter system is stable and the states are attracted to the surface.

\subsubsection{Simulation results for the sloped reference surface}

A simulation for the system with sloped reference surface is done in MATLAB/Simulink. The system parameters selected are shown in Table 1. The objective is to drive the input current and output voltage state trajectories to the equilibrium point of the surface shown in Fig. 14. The ADC delay is $2.5 \mu \mathrm{s}$, its default value, which generates the hysteresis desired by the controller.

The simulation results are shown in Fig. 16 and Fig. 17. Fig. 16 shows the variation of the input current and output voltage states over time. It may 

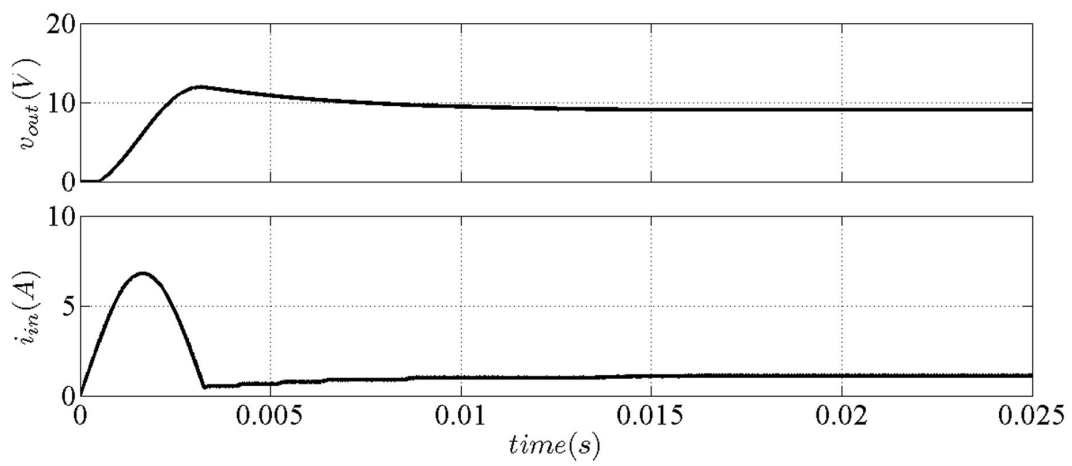

Figure 16: Time domain plot for the digital sliding-mode control strategy for a sloped reference surface in a dc-dc boost converter.

be noted that the input current and output voltage states reach the desired equilibrium point from a cold start thereby validating the control strategy. Fig. 17 shows the state-plane plot of the output voltage and input current states along with the reference sliding surface. The control strategy drives the state trajectories to the surface, compels the states to stay on the surface and drives them to the desired equilibrium point.

\subsubsection{Experimental results for the sloped reference surface}

The system parameters in Table 1 are used in an experimental set-up to validate the control strategy in real-time hardware. The sloped surface shown in Fig. 14 is programmed into 16384 memory locations of the EEPROM and the $\mathrm{ADC}$ is set for a propagation delay of $2.5 \mu \mathrm{s}$. In this set up, 14 input pins of the EEPROM are used to accommodate the signals coming from the two ADCs. Fig. 18 shows the variation of the input current, output voltage 


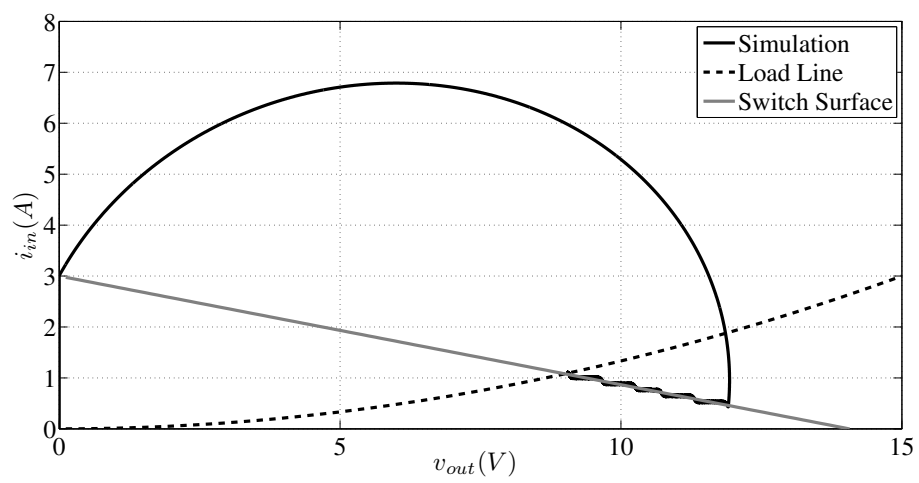

Figure 17: State-plane plot for the digital sliding-mode control strategy for a sloped reference surface in a dc-dc boost converter.

and switch states with time. It may be noted that the output voltage finally reaches a value of around $9 \mathrm{~V}$ and the input current reaches an average value of around 1.20 A from a cold start, which is close to the desired equilibrium point values, thereby experimentally validating the control strategy. Fig. 19 shows the state trajectories in the steady state, indicating that they have reached values close to the equilibrium point. Fig. 20 shows the stateplane plot obtained from the experimental data. This figure shows that the controller drives the system state trajectories to the required sliding surface and finally to the equilibrium point. The deviation from the simulation results may be attributed to the various losses in the hardware, signal delays other than the ADCs in the circuit and the ADC output resolution. In Fig. 18, it may be observed that the input current trajectory never crosses a value of $5.8 \mathrm{~A}$. This is due to a hardware limitation for this work, as discussed in Section 6.1.3. Hence, the sloped surface can be successfully implemented as a reference surface in the memory chip and the digital sliding- 


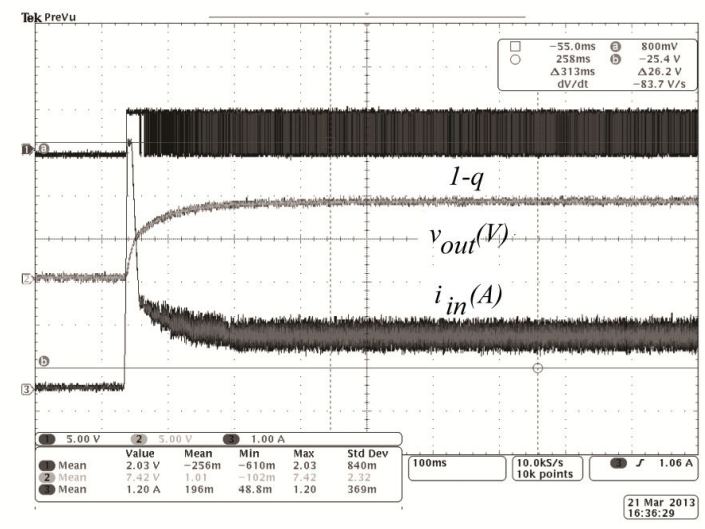

Figure 18: Experimental time domain plot for the digital sliding-mode control strategy for a sloped reference surface in a dc-dc boost converter. Scale Factors: $5 \mathrm{~V} /$ div for $v_{\text {out }}$, $5 \mathrm{~V} /$ div for $1-\mathrm{q}, 1 \mathrm{~A} / \mathrm{div}$ for $i_{\text {in }}$ and $100 \mathrm{~ms} / \mathrm{div}$ for the time scale

mode hysteretic controller could be designed effectively such that the states reach the reference surface, slide along it and reach the desired equilibrium point.

\section{Discussion}

This work implemented a digital hysteretic sliding-mode control technique in an EEPROM. Linear surfaces in the state plane are used as reference surfaces in the memory chip. The digital sliding-mode hysteretic controller could be effectively designed such that the states slide along the reference surfaces and reach the desired equilibrium points.

The selection of the size of the ADC and the memory size of the EEPROM is dependent on the resolution necessary for proper implementation of the 


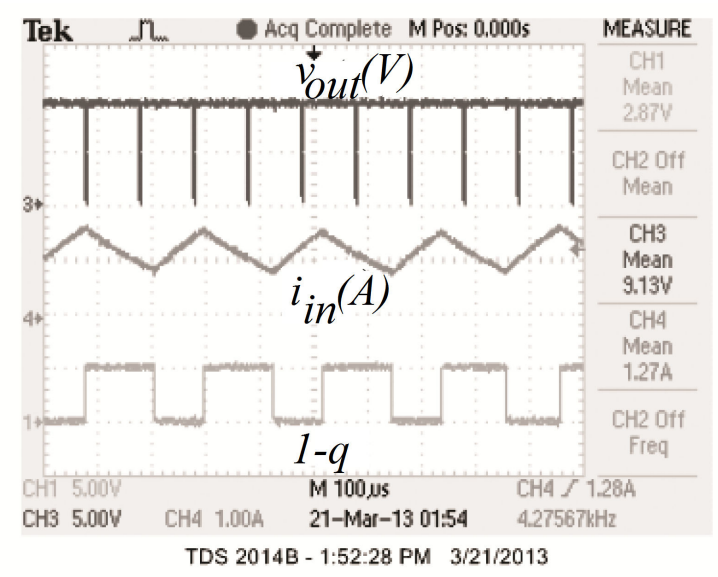

Figure 19: Experimental time domain plot in the steady state for the digital sliding-mode control strategy for a sloped reference surface in a dc-dc boost converter.

sliding-mode controller in the example systems chosen. Higher the resolution, better the control. There exists an upper limit to the resolution based on the size of the memory chip chosen for the design and the resolution of the ADC. The limits of ADC resolution depend on the number of ADC output bits. The choice of the memory size of the chip depends on the numbers of state signals required and the $\mathrm{ADC}$ resolution.

The control strategy is made simpler in terms of hardware design by eliminating the switch state feedback to the controller to generate hysteresis. The ADC signal propagation delay generates the required hysteresis band. There are both upper and lower bounds to the delay in the ADC, beyond which this strategy will cease to work. If the delay is made very small, the hysteresis band will become extremely small and result in very high switching frequency. On the other hand, if the delay is very big, then the memory table control logic will react to a very delayed signal and output an erroneous 


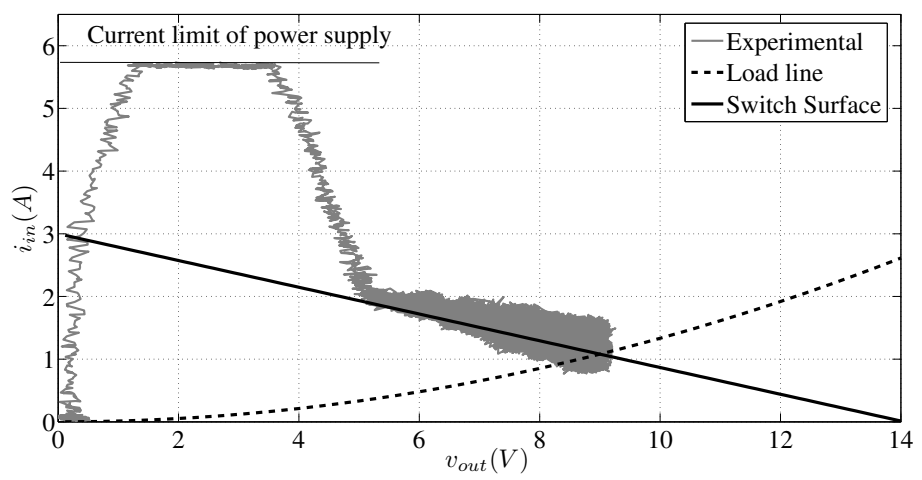

Figure 20: Experimental state-plane plot for the digital sliding-mode control strategy for a sloped reference surface in a dc-dc boost converter.

switch state. Fig. 3 and Fig. 4 illustrate that the switching logic is developed in such a way that the system states are confined within a hysteresis band. This ensures that the switching frequency is a finite number and the switching control signal is a deterministic one that limits the chattering in the neighborhood of the switching surface.

The investigation on the stability of the chosen surfaces pertaining to the example systems is a very generic method of stability analysis. This method shows that the surfaces chosen are stable for the example systems considered. This analysis is a guideline for any surface stability analysis for a power electronic device based system.

This control strategy is implemented in a dc-dc boost converter. This method is equally applicable for any converter topology and also for systems having multiple power electronic converters. A very important criteria for this strategy to work is the selection of a stable sliding surface pertaining to the system of choice. If the surface is stable for the example system, then 
this strategy can be implemented in an exact similar manner.

In this work, the stored surfaces are constructed for specific discrete scenarios. If there is any transient event, such as a step change in load, the surface will change. It is therefore necessary to store the new surface in the EEPROM prior to the run. Many different scenarios may be thought of based on the system operation and different surfaces may be stored and different switching logic may be generated using the method shown in this paper for these different scenarios. An additional bit of the EEPROM will be necessary to pass on the information for these events. In the situation of a contingency that is not explicitly used to derive the surfaces, if it lies within the event space used for the derivation, it will treat the closest scenario as the reference because of the quantization effects of the digital controller used. Hence, the calculations are optimal for specific cases, but are still viable for other cases. Experimental results for these types of transient events and how this controller can work during those events have been shown in [15].

If the number of scenarios increase, the computational burden of the controller becomes extensive. The larger the event space, the more extensive the computational burden is. However, all the calculations for the generation and storage of the surface are done off-line. The solution to the off-line computation is stored in the EEPROM memories prior to the system operation. During the system operation, the EEPROMs act as memory lookup tables and control the state trajectories. Therefore, there is no computation involved during the operation.

For the constant current reference surface as shown in Fig. 6, a combined Pulse-width modulated and Proportional-Integral (PWM-PI) controller is 
capable of doing the same thing as the proposed controller. However, for a change in the system parameter or due to any transient condition, such as a step change in the load, the gains for the PI controller need to be tuned. For the proposed digital sliding mode hysteretic controller, no modification is necessary due to any change in system parameter or transients. This controller is self adjusting and the controller objective will be achieved without any external involvement. When the reference surface becomes a little more complicated, such as the sloped surface shown in Figures 3, 4 and 14, the implementation of the PWM-PI controller becomes even more complicated. A variable time-dependent duty cycle generation becomes necessary based on the expected state performance. The PWM-PI controller becomes even more complex if the surface becomes non-linear and it may not even be possible to design a PWM-PI controller for such complex surfaces. However, the controller developed in this work, is capable of tracking and following any complex linear or non-linear surface. The linear or non-linear surface needs to be stored as reference surface in the EEPROM and the switching logic will generate the required switch pattern to control the converter. It may be noted that if there is only one steady state operating point, a straight line surface would suffice. However, there are occasions that a sloped or curved surface can improve dynamic response or implement a desired function [30]. Moreover, a sliding mode controller with a curved surface provides a faster response to any type of perturbation while a PI controller is capable of only rejecting small-signal perturbations. One of the primary advantages of a sliding mode controller over a PI controller is that the former is a faster control method and since discontinuous control signals are allowed, a direct control 
of the switch state is possible.

\section{Future Work}

The calculations for the examples shown in this work are optimal for these specific cases, but are still viable for other cases. Further work is needed to determine when a specific solution space will cover all contingencies with a higher level of confidence. This is not unlike neural networks, where the systems is trained on a specific set of cases and is able to extrapolate to cases outside the training set. It is advisable to think of as many situations as possible before the problem formulation. This will ensure that the scenarios cover almost all the situations pertaining to the system operation.

In this work the limits of the memory size has not been explicitly derived. There is scope to formulate a mathematical formula that would explicitly specify the upper and lower limits of the memory resolution for a particular system.

Similar limits for the ADC latency may also be derived.

The derivation of a possible mathematical relationship between the ADC latency and the hysteresis band, and hence the switching frequency, has been left for future work.

Relationship between the hysteresis and the errors in the switch state need to be analyzed for better performance.

\section{Conclusions}

The contribution of this work is the development of a fast, low-cost and self adjusting robust controller that can be implemented for any converter 
topology and any reference surface. The examples are shown for a dc-dc boost converter and a straight line and a sloped surface. However, the method can be used for any other converter topology and other surfaces. The proposed strategy effectively controls the system for the desired reference surfaces and desired system responses, as shown in the simulation and experimental results. It is also observed that since the real time implementation of this strategy does not require any on site mathematical calculations, the control action takes place in a very little time. EEPROM memory chips are readily available, robust and low-cost compared to any other controllers capable of performing a similar operation, make this strategy a low cost and effective control strategy for dc-dc converters.

\section{Acknowledgment}

This material is based upon work supported by the National Science Foundation under Grant No.0901094.

\section{References}

[1] X. Liu, P. Wang, P. C. Loh, A hybrid ac/dc microgrid and its coordination control, IEEE Transactions on Smart Grid 2 (2) (2011) 278-286.

[2] H. Guldemir, Sliding mode control of dc-dc boost converter, Journal of Applied Sciences 5 (3) (2005) 588 -592.

[3] J. H. Su, J. J. Chen, D. S. Wu, Learning feedback controller design of switching converters via MATLAB/SIMULINK, IEEE Transactions on Education 45 (4) (2002) 307 - 315. 
[4] P. Mattavelli, L. Rossetto, G. Spiazzi, Small-signal analysis of dc-dc converters with sliding mode control, in: Proceedings of Applied Power Electronics Conference and Exposition, Vol. 1, 1995, pp. 153 -159.

[5] H. Sira-Ramirez, Sliding motions in bilinear switched networks, IEEE Transactions on Circuits and Systems 34 (8) (1987) 919-933.

[6] A. Dashtestani, B. Bakkaloglu, A fast settling oversampled digital sliding-mode dc-dc converter, IEEE Transactions on Power Electronics 30 (2) (2015) 1019-1027.

[7] Y. Tarte, Y. Q. Chen, W. Ren, K. Moore, Fractional horsepower dynamometer - a general purpose hardware-in-the-loop real-time simulation platform for nonlinear control research and education, in: Proceedings of the IEEE Conference on Decision and Control, 2006, pp. 3912-3917.

[8] J. Alvarez-Ramirez, G. Espinosa-Perez, D. Noriega-Pineda, Currentmode control of dc-dc power converters: a backstepping approach, in: Proceedings of the IEEE International Conference on Control Applications, 2001, pp. $190-195$.

[9] H. Sira-Ramirez, Nonlinear variable structure systems in sliding mode: the general case, IEEE Transactions on Automatic Control 34 (11) (1989) 1186-1188.

[10] H. Guldemir, Study of sliding mode control of dc-dc buck converter, Journal of Energy and Power Engineering 3 (2011) 401 -406. 
[11] Y. B. Shtessel, A. S. I. Zinober, I. A. Shkolnikov, Boost and buckboost power converters control via sliding modes using dynamic sliding manifold, in: Proceedings of the Conference on Decision and Control, Vol. 3, 2002, pp. 2456-2461.

[12] Y. He, F. Luo, Sliding-mode control for dc-dc converters with constant switching frequency, Control Theory and Applications 153 (1) (2006) 37 $-45$.

[13] B. Banerjee, W. W. Weaver, Geometric manifold control of power electronics in dc microgrids, in: Proceedings of the IEEE Workshop on Control and Modeling for Power Electronics, 2012, pp. 1 -8.

[14] A. Cid-Pastor, L. Martinez-Salamero, A. E. Aroudi, R. Giral, J. Calvente, R. Leyva, Synthesis of loss-free resistors based on sliding-mode control and its applications in power processing, Control Engineering Practice (2013) 689-699.

[15] B. Banerjee, W. W. Weaver, Generalized geometric control manifolds of power converters in a de microgrid, IEEE Transactions on Energy Conversion 29 (4) (2014) 904-912.

[16] Y. F. Liu, L. Jia, Performance enhancement with digital control technologies for dc-dc switching converters, in: Proceedings of the IEEE Control and Modeling for Power Electronics, 2010, pp. 1 -8.

[17] M. Marwali, A. Keyhani, Control of distributed generation systems-part I: Voltages and currents control, IEEE Transactions on Power Electronics 19 (6) (2004) 1541-1550. 
[18] O. König, G. Gregorciiĉ, S. Jakubek, Model predictive control of a dcdc converter for battery emulation, Control Engineering Practice (2013) 428-440.

[19] G. Pitel, P. Krein, Minimum-time transient recovery for dc-dc converters using raster control surfaces, IEEE Transactions on Power Electronics 24 (12) (2009) $2692-2703$.

[20] J. M. Pereira, O. Postolache, F. Dengchao, V. Viegas, P. Girao, Advantages of pwm-a/d conversion techniques in smart sensing systems, in: Proceedings of the Conference on Electronic Measurements and Instruments, 2007, pp. 5-10.

[21] B. Kumari, R. S. Rana, C. Y. Patil, Fpga and dspace based sliding mode control of boost converter for pem fuel cell application, International Journal of Computer Applications 51 (18) (2012) 4-10.

[22] J. Q. Borrás, Mcu controlled dc-dc buck/boost converter for super capacitors, Master's thesis, KTH Royal Institute of Technology, Stockholm, Sweden (6 2012).

[23] N. Gupta, S. P. Singh, S. P. Dubey, Dsp based adaptive hysteresis-band current controlled active filter for power quality conditioning under nonsinusoidal supply voltages, International Journal of Engineering, Science and Technology 3 (4) (2011) 236-252.

[24] http://www.digikey.com/, accessed: 04-16-2016, 2-42 PM.

[25] https://estore.ti.com/, accessed: 04-16-2016, 2-45 PM. 
[26] N. H. Kutkut, C. Q. Lee, I. Batarseh, A generalized program for extracting the control characteristics of resonant converters via the state-plane diagram, in: Proceedings of the IEEE Power Electronics Specialists Conference, Vol. 2, 1995, pp. 1236-1242 vol.2.

[27] Y. Li, Y. Chen, I. Podlubny, Stability of fractional-order nonlinear dynamic systems: Lyapunov direct method and generalized mittag-leffler stability, Computers and Mathematics with Applications 59 (2010) $1810-1821$.

[28] B. Banerjee, Local digital control of power electronic converters in a dc microgrid based on a-priori derivation of switching surfaces, Ph.D. thesis, Michigan Technological University, Houghton, Michigan, U.S.A., http://digitalcommons.mtu.edu/etds/651 (12 2013).

[29] S. A. Bock, J. Pinheiro, H. Grundling, H. Hey, H. Pinheiro, Existence and stability of sliding modes in bi-directional dc-dc converters, in: Proceedings of the IEEE Power Electronics Specialists Conference, Vol. 3, 2001, pp. 1277-1282.

[30] W. W. Weaver, P. T. Krein, Optimal geometric control of power buffers, IEEE Transactions on Power Electronics 24 (5) (2009) 1248 -1258. 